\title{
Acendendo as Luzes: uma inovação no Cuidado a Saúde dos Pacientes Oncológicos, Familiares e Equipe
}

\section{Gelvani Locateli \\ GMestranda em Ciências da Saúde pela Universidade Comunitária da Região de Chapecó (UNOCHAPECÓ)-Campus Chapecó \\ Natanael Chagas \\ Cirurgião-Dentista pela Universidade Comunitária da Região de Chapecó (UNOCHAPECÓ)-Campus Chapecó. \\ Caroline Menzel Gato \\ Acadêmica do curso de graduação em Enfermagem na Universidade Federal da Fronteira Sul (UFFS) - Campus Chapecó.}

\section{Gabriela Gonçalves de Oliveira}

Doutora em Patologia Experimental. Docente da Universidade Federal da Fronteira Sul (UFFS).

\section{Leoni Terezinha Zenevicz}

Doutora em Gerontologia Biomédica. Docente da Universidade Federal da Fronteira Sul (UFFS).

\section{Resumo}

As práticas Integrativas e complementares têm se incorporado ao sistema de saúde de maneira significativa, na assistência a saúde dos pacientes, especialmente na área da oncologia. O câncer é uma doença devastadora que impacta de maneira negativa na vida do paciente, família e equipe. A utilização das Praticas Integrativas e Complementares (PIC's) são uma alternativa que complementam os tratamentos convencionais na melhora da qualidade de vida e no cuidado a dimensão espiritual do ser humano.

Objetivo: Relatar a experiência de implantação das Terapias Integrativas e Complementares através do Projeto de Extensão Luzes.

Métodos: As atividades realizadas são Reiki, Auriculoterapia, massagem laboral, meditação, grupo de oração, música e canto, Bio Energetics Medicine, Contação de histórias, confecção de origamis, avaliação da saúde bucal, construção de Mandalas e pinturas. As PIC's são desenvolvidas por um grupo de 136 voluntários dentre docentes, discentes e profissionais de diferentes áreas e da comunidade em geral. Desde sua implantação em Agosto de 2018 já atendeu mais de 1700 pessoas. É desenvolvido dentro do Hospital Regional do Oeste de Chapeco SC nos setores de Oncologia, Quimioterapia e Radioterapia.

Resultados: As terapias integrativas e complementares são reconhecidas como benéficas pelos pacientes, familiares e profissionais. Consideradas aliadas na melhora da qualidade de vida durante o período de 
internação e tratamento. Também citada como uma maneira diferenciada de assistência a dimensão espiritual porque propicia a formação de vinculos mais empáticos entre profissionais, pacientes e família. Observa-se também uma maior qualificação no processo de formação profissional dos acadêmicos dos cursos de Enfermagem e Medicina da UFFS.

Conclusões: A utilização das praticas integrativas e complementares no ambiente hospitalar foram sem sombra de dúvidas um desafio. Devemos reconhecer que valeu o desafio de implantar as Terapias Integrativas e Complementares que aliadas ao tratamento convencional trouxeram benefícios impares a tríade paciente, família e profissional. Um espaço de prática das PIC's possibilita a formação de recursos humanos voltados ao SUS, com uma visão ampliada ao processo saúdedoença e mais competentes e comprometidos com a saúde integral do ser humano.

Palavras-chave: Enfermagem; PIC's; Oncologia; Profissionais de Saude.

\section{Abstract}

Integrative and complementary practices have been significantly incorporated into the health system in the health care of patients, especially in the area of oncology. Cancer is a devastating disease that negatively impacts the lives of patients, families and staff. The use of Integrative and Complementary Practices (ICPs) is an alternative that complements conventional treatments in improving the quality of life and caring for the spiritual dimension of the human being.

Objective: Report the experience of implementation of Integrative and Complementary

\section{Introdução}

Segundo a Organização Mundial da Saúde (OMS), estima-se para o ano de 2025, uma incidência de 20 milhões de casos de câncer
Therapies through the Lights Extension Project.

Methods: The activities performed are Reiki, Auriculotherapy, Labor Massage, Meditation, Prayer Group, Music and Singing, Bio Energetics Medicine, Storytelling, Origami Making, Oral Health Assessment, Mandala Construction and Painting. PICs are developed by a group of 136 volunteers including teachers, students and professionals from different fields and the wider community. Since its implementation in August 2018, it has served more than 1700 people. It is developed within the Western Regional Hospital of Chapeco SC in the Oncology, Chemotherapy and Radiotherapy sectors.

Results: Integrative and complementary therapies are recognized as beneficial by patients, families and professionals. Considered allies in improving the quality of life during hospitalization and treatment. Also cited as a different way of assisting the spiritual dimension because it provides the formation of more empathic links between professionals, patients and family. There is also a higher qualification in the process of professional training of students of Nursing and Medicine courses at UFFS.

Conclusions: The use of integrative and complementary practices in the hospital environment was undoubtedly a challenge. We must recognize that the challenge of implementing the Integrative and Complementary Therapies that combined with the conventional treatment brought unfair benefits to the patient, family and professional triad was worth the challenge. A practice space for ICPs enables the formation of human resources focused on the SUS, with a broader view of the health-disease process and more competent and committed to the integral health of human beings.

Keywords: Nursing; PIC's; Oncology; Health professionals. em todo o mundo, segundo a segunda causa de mortes por doenças crônicas no Brasil $(16,3 \%)^{1}$ 
O câncer é uma enfermidade que na maioria dos casos desencadeia sofrimento físico e emocional aos pacientes, levando a redução da autoestima frente às mudanças que decorrem do processo de adoecimento. A enfermidade desperta sentimentos de tristeza, angústia e medo, os quais precisam ser combatidos tanto pelo paciente como pela família que o acompanha. As etapas vivenciadas ao longo do tratamento pelos familiares de pessoas com câncer, os tornam mais vulneráveis devido as imensos desafios em decorrência da doença. ${ }^{2}$

A espiritualidade e a conexão com o poder supremo na busca das respostas as questões essenciais da vida humana, e se traduz pelo equilíbrio, amor, paz, compaixão, reconexão e essência. Considerada uma ferramenta que afeta sobremaneira o enfrentamento dos pacientes oncológicos, ressignificando a doença, melhorando a qualidade de vida e diminuindo o tempo de hospitalização. Para Reginato mais do que um novo conhecimento, a espiritualidade é uma forma de ver o contexto dos acontecimentos através de uma nova perspectiva, a qual por muito tempo foi apenas reduzida a uma visão focada na parte técnica, e que na atualidade se abre para a reflexão acerca das questões essenciais e existenciais. ${ }^{3}$

\section{O Brasil oferece vinte e nove práticas} integrativas pelo SUS e esta se tornando um país líder na oferta dessa modalidade de assistência na atenção básica. ${ }^{4} \quad \mathrm{~A}$ assistência espiritual, vem ocupando espaços pela academia cientifica em diferentes contextos do ensino, da pesquisa e da extensão, porque além de ser um construto da vida humana, enfoca elementos inatingíveis que conferem vitalidade e significado aos eventos que ocorrem na vida. A musicoterapia, acupuntura e massagem são as práticas usadas com maior frequência nos cuidados paliativos, sendo aliadas ao tratamento convencional. ${ }^{5}$

A Oncologia Integrativa, apesar de precoce em nosso país, propõe essa combinação das práticas integrativas e complementares com o cuidado tradicional, de modo a resgatar os princípios da bioética, procurando evitar danos e respeitando a autonomia do paciente enquanto busca-se estratégias para contribuir com seu bem-estar e o estar bem. ${ }^{6,7}$

Dessa forma, este trabalho tem por finalidade discorrer acerca da criação e implantação do projeto de extensão "Cuidados Paliativos e Espiritualidade: Linhas Entrelaçadas no Cuidado ao Paciente Oncológico, denominado: Projeto Luzes". Vinculado à Universidade Federal da Fronteira Sul - Chapecó, o projeto objetiva cuidar da dimensão espiritual do paciente, família e equipe através do uso das Praticas Integrativas e Complementares (PIC's).

\section{Métodos}

O local selecionado para desenvolvimento das atividades relativas ao projeto é o Hospital Regional do Oeste (HRO) localizado na cidade de Chapecó-SC, Restringindo-se a princípio, as ações ao setor de Oncologia. O público alvo é 
formado por pacientes, acompanhantes e funcionários conforme demanda espontânea. Desde a sua implantação em Agosto de 2018, 87 pessoas foram atendidas diariamente, totalizando 1740 atendimentos/mês.

Anteriormente ao início do projeto no hospital, realizou-se uma sensibilização com todos os voluntários com o objetivo de abordar a temática do câncer e contextualizar a rotina vivenciada por estes pacientes. Nesta ocasião também foi abordado higienização das mãos tão necessária para evitar infecções cruzadas ao adentrarmos com o projeto e o contato com os pacientes oncológicos. Ministrado por uma psicóloga e docente da universidade, a integração foi necessária, pois, muitos integrantes do projeto nunca haviam tido nenhum tipo de contato com a doença e/ou com o paciente oncológico, além das inseguranças e ansiedades provocadas pelo adentrar no ambiente hospitalar.

Atualmente, o projeto conta com um número aproximado de 136 voluntários, entre docentes e discentes Universidade Federal da Fronteira Sul(UFFS) e Universidade do Desenvolvimento de Santa Catarina(UDESC), além de profissionais da saúde e da comunidade externa, de diferentes setores, além da parceria com os voluntários do movimento "Eu sou a Paz".

As atividades implantadas atualmente são: o Reiki, Auriculoterapia, massagem laboral e massoterapia, meditação, Bio Energetics Medicine, grupo de oração, música e canto,
Contação de histórias, construção de origamis, pinturas de Mandalas e avaliação da saúde bucal. Para o desenvolvimento das ações dividiuse os voluntários em subgrupos de acordo com as afinidades de cada voluntário com as dinâmicas escolhidas, optando-se por deixar um docente da universidade responsável por cada um dos subgrupos. Cada voluntário disponibiliza 4 horas para atuar dentro do projeto.

O Reiki e a Auriculoterapia são práticas inclusa na Política Nacional de Práticas Integrativas e Complementares (PNPIC) no SUS, e são desenvolvidas numa sala especialmente organizada para desenvolvimento destas atividades e localiza no terceiro andar do hospital que abraçou o projeto, ou seja, a ala da Oncologia.

O atendimento é realizado por uma dupla de voluntários que prestam assistência para no máximo dois pacientes por vez, devido à disponibilidade de macas na sala.

Os voluntários que trabalham com a massagem realizam a prática em um espaço do hospital designado para descanso e demais atividades. Nesse local estão localizadas as cadeiras de massagem as quais foram conquistadas através de doações para o projeto. A utilização delas proporciona a expansão do atendimento do projeto para os funcionários de todas as unidades e setores do HRO.

O grupo de oração trabalha através da leitura de mensagens e orações variadas sempre 
respeitando a religião e preferências de cada paciente. Os voluntários desenvolvem a ação nas enfermarias da unidade de Oncologia e nos setores de quimioterapia e radioterapia. A Contação de histórias decorre-se de forma semelhante, com o preceptor convidando os pacientes e acompanhantes que demonstrem interesse em participar.

Os voluntários do movimento "Eu sou a paz" trabalham com a meditação, construção de origamis e pinturas de Mandalas. A proposta se desenvolve sempre na terceira quinta-feira de cada mês quando os voluntários dirigem-se a unidade e convidam os pacientes e acompanhantes para participar das dinâmicas.

A musicoterapia é trabalhada com instrumentos como violão e violino, além do canto, sendo que os voluntários passam nas enfermarias oferecendo o momento da música aos pacientes e familiares. Quando possível realiza-se a música no corredor da unidade, proporcionando um momento de alegria e descontração também aos demais acompanhantes e profissionais da unidade. $\mathrm{O}$ projeto conta com dois grupos designados para a música formados por acadêmicos dos cursos de Enfermagem e Medicina da UFFS.

Em reuniões realizadas com o todo o grupo, desenvolveu-se o cronograma de atividades vigente até o final de 2020. As ações ocorreram de segunda a sexta feira de acordo com a conveniência dos voluntários e suas equipes de trabalho, durante os períodos matutino, vespertino e noturno. Nos sábados são realizadas atividades lúdicas com brincadeiras e jogos. A atividade lúdica cria novas relações com a vida, estabelece outros jeitos de ver e viver o internamento hospitalar, permeadas pela amizade, cooperação, solidariedade, amorosidade e senso de responsabilidade coletiva. São responsáveis pela atividade acadêmicos de enfermagem, medicina e pedagogia da UFFS e UDESC.

A Saúde Bucal é trabalhada de modo a prevenir e orientar afecções bucais. Como o público alvo são pacientes na Oncologia, diversas patologias orais são oriundas das medicações utilizadas pelo paciente. Outro fator importante é que os pacientes que realizam seu tratamento tem seu nível de imunidade comprometido, sendo outra causa de patologias bucais. Portanto, pacientes oncológicos são extremamente suscetíveis à patologias bucais. Os trabalhos desenvolvidos são a higienização das próteses, avaliação da condição oral, entrega de creme dental e escova dental, doados pelo Centro de Especialidade Odontológica (CEO) da Secretaria Municipal de Saude. A coordenação da equipe e realizada por uma odontóloga e discentes da área.

\section{Resultados}

Desde que o projeto foi implantado, observouse um aumento crescente da participação de docentes e discentes de seis diferentes cursos da Universidade Federal da Fronteira Sul (Tabela 1). Formou-se uma rede de 136 parceiros de 
diferentes setores da sociedade constituindo uma rede de solidariedade com uma dimensão nunca antes imaginada. As Terapias Integrativas e Complementares são ainda incipientes nas instituições hospitalares do Oeste de Santa Catarina e este trabalho pode servir de exemplo e de inovação a outros serviços de atenção a saúde do paciente oncológico, familiares e equipe. As terapias integrativas e complementares associadas ao tratamento convencional possibilitam uma forma de cuidar do ser humano doente e não da doença, assim contemplando a dimensão espiritual, até então desconsiderada no cuidado. Aos familiares um momento reequilibrar as energias para os dias vindouros e aos profissionais o cuidado a saúde física e mental, especialmente do estresse advindos das longas jornadas em contato com o sofrimento humano.

\section{Discussão}

O projeto Luzes vem ao encontro das diretrizes apontadas pela Política Nacional de Práticas Integrativas e Complementares (PNPIC) que assinalam a necessidade de uma assistência integral com abordagem holística a saúde de pacientes portadores de doenças incuráveis e progressivas como o câncer.

As Terapias Integrativas e Complementares (PIC's) utilizadas neste projeto romperam uma história centrada no combate das doenças passando a uma assistência a integralidade do homem doente, fomenta pela empatia, o respeito e a autonomia.
As ações realizadas pelo projeto foram consideradas positivas pelos pacientes, familiares e equipe sendo sugerida sua ampliação para outros setores da instituição.

A espiritualidade é uma dimensão importante e inata do ser humano e fator de bem-estar, conforto e esperança, que precisa ser incluída no processo de humanização dos cuidados em saúde. Portanto, cuidar desta dimensão é uma prioridade com impactos significativos na saúde integral das pessoas e as PIC's são uma possibilidade real de suprir esta necessidade humana básica. Concluímos que aliar essas práticas nos cuidados hospitalares possibilitam ao paciente conforto, forças para o enfrentamento da doença, melhor adesão ao tratamento, conforto nas perdas e finitude.

\section{Considerações finais}

A espiritualidade e as práticas integrativas e complementares ainda são temas negligenciados de maneira geral nos cursos da área da saúde, ainda que nos últimos anos tenha-se ampliado as discussões sobre sua importância e sua abrangência inclusive no Sistema Único de Saúde.

Também destacamos o protagonismo do projeto de extensão LUZES na assistência espiritual dos pacientes oncológicos dentro da maior instituição hospitalar da região Oeste de Santa Catarina.

O projeto demonstra a importância da interação ensino serviço para a formação de 
recursos humanos para o SUS, numa perspectiva de profissionais de saúde mais comprometidos e competentes na assistência do ser humano como um todo.

\section{Referências}

${ }^{1}$ Sousa FFPRD, Freitas SMFM, Farias AGS, Cunha MCSO, Araújo MFM, Veras VS. Enfrentamento religioso/espiritual em pessoas com câncer em quimioterapia: revisão integrativas da literatura. SMAD. 2017, 13(1): 45-51.

${ }^{2}$ Mattos K, Blomer TH, Campos ACBF, Silverio MRegina.Estratégias de enfrentamento do câncer adotadas por familiares de indivíduos em tratamento oncológico. Rev. Psicol. Saúde [online]. 2016, 8(1): 01-06.

${ }^{3}$ Reginato V, Benedetto MAC, Gallian DMC. Espiritualidade e saúde: uma experiência na graduação em medicina e enfermagem. Trab. Educ. Saúde, 2016, 14(1): 237-55.

${ }^{4}$ BRASIL. Ministério da Saúde. Acesso em 15/01/2019. Disponível em: http://portalms.saude.gov.br/noticias/agenciasaude/42737-ministerio-da-saude-inclui-10-novas-praticas-integrativas-no-sus.

${ }^{5}$ Caires JS, Andrade A, Amaral JB, Calasans MTA, Rocha MDS. A utilização das terapias complementares nos cuidados paliativos: benefícios e finalidades. Cogitare Enferm. 2014; 19(3): 14-20.

${ }^{6}$ Siegel P, Barros NF. O que é Oncologia Integrativa? Cad. Saúde Coletiva. 2013; 21(3): 348-54.

${ }^{7}$ Siegel P, Barros NF. Por que as pesquisas em Oncologia Integrativa são importantes? Rev. Bras. Cancerologia. 2013; 59(2): 249-53.

${ }^{8}$ Seki NH, Galheigo SM. O uso da música nos cuidados paliativos: humanizando o cuidado e facilitando o adeus. Interface. 2010; 14(33): 273-84.

${ }^{9}$ Silva DIS. Significados e práticas da espiritualidade no contexto dos cuidados paliativos em pacientes oncológicos adultos. Rev HCPA. 2011; 31(3): 353-8.

${ }^{10}$ Evangelista CB, Lopes MEL, Costa SFG, Batista PSS, Batista JBV, Oliveira AMM. Cuidados paliativos e espiritualidade: revisão integrativa da literatura. REBEn. 2016; 69(3): 554-63.

${ }^{11}$ Peres MFP, Arantes ACLQ, Lessa PS, Caous CA. A importância da integração da espiritualidade e da religiosidade no manejo da dor e dos cuidados paliativos. Rev Psiq Clín. 2007; 34(1): 82-7.

${ }^{12}$ BRASIL. Ministério da Saúde. Secretaria de Atenção à Saúde. Departamento de Atenção Básica. Política Nacional de Práticas Integrativas e Complementares no SUS - PNPIC-SUS. Brasília: Ministério da Saúde, 2006. 
Tabela 1. Cursos de graduação envolvidos no projeto Luzes e respectivas atividades realizadas.

\begin{tabular}{|l|l|}
\hline \multicolumn{1}{|c|}{ Curso } & \multicolumn{1}{c}{ Atividades } \\
\hline Medicina & Música e canção \\
\hline \multirow{2}{*}{ Enfermagem } & Grupo de Oração \\
\hline & Coral \\
\hline & Auriculoterapia \\
\hline Psicologia & Grupo de Oração \\
\hline Jisioterapia & Jogos e Atividades Festivas \\
\hline Confecção de origami e pintura de mandala \\
\hline Odontologia & Contação de história musicada \\
\hline Direito & Auriculoterapia \\
\hline Acupuntura \\
\hline Profissionais já formados de & Avaliação e orientações de saúde bucal \\
\hline diferentes áreas e voluntários & Esclarecimento sobre os direitos do \\
\hline
\end{tabular}

Submissão: $22 / 02 / 2019$

Aceite: 06/03/2020 